\title{
PRESSURE MEASUREMENTS ON THE GATE SUBJECTED TO SUBMERGED HYDRAULIC JUMP
}

\author{
SOCHHEAT SMOK \& ENDER DEMIREL \\ Department of Civil Engineering, Eskisehir Osmangazi University, Turkey
}

\begin{abstract}
Vertical gates are commonly used to control the flow and to measure the discharge in irrigation canals and natural streams. Submerged hydraulic jump forms downstream of a gate when the tail water depth is greater than the conjugate depth of the free jump. Interaction of the recirculating flow with the incoming flow with high momentum creates surface pressures fluctuations on the gate. In this study, experimental studies were carried out in a laboratory flume under different flow conditions of jet Froude number and submergence factor in order to provide a clear understanding of how the vortex induced pressure fluctuations affect the gate stability. Simultaneous pressure measurements were made using pressure sensors attached to the different locations on the gate lip. Spatial variations of time-averaged and instantaneous pressure coefficients at the gate lip were evaluated for different inlet Froude numbers and submergence factors. Although the submergence factor is not very effective on the distribution of mean pressure coefficient, Froude number can significantly affect the mean pressure distributions. On the other hand, spatial distributions of instantaneous pressure coefficient strongly depend on both jet Froude number and submergence factor. Frequency spectra of the lift pressures reveal that the magnitude of the spectra increases as the Froude number increases and the slope of the spectra is independent of both Froude number and submergence factor.
\end{abstract}

Keywords: submerged jet, pressure measurement, lift pressure, vertical gate.

\section{INTRODUCTION}

Spillways and weirs are the common hydraulic structures to regularize and measure the flow in streams depending on the seasonal variations in flow conditions. Such water structures significantly alter the flow conditions since the solid structure covers the entire cross section of the channel. Turbulent flow creates complex flow structure which may result in vortical flows in the vicinity of the control structure and drastic increase in drag and lift pressures on the walls. Vortex induced hydrodynamic pressures acting on the structure may give rise to oscillate and breakdown of the structure depending on the flow conditions. Vertical sluice gates have been used in hydraulic engineering applications in the last decade due to its multipurpose uses in rivers and irrigation canals. Vertical gates causes the flow regime to become supercritical at the entrance and consequently hydraulic jump forms downstream of the gate. As the tail water depth becomes greater than the conjugate depth of the free-jump, hydraulic jump occurs beneath the free-surface and this type of jump is classified as submerged hydraulic jump (SHJ) [1]. Degree of the submergence can be assessed according the following relation [2]

$$
S=\left(y_{t}-y_{2}\right) / y_{2}
$$

where $y_{t}$ is the tail water depth and $y_{2}$ is subcritical conjugate depth of the gate opening, which can be calculated from the following well known Belanger's equation:

$$
y_{2}=0.5 y_{1}\left(\sqrt{1+8 F_{1}^{2}}-1\right) \text {. }
$$




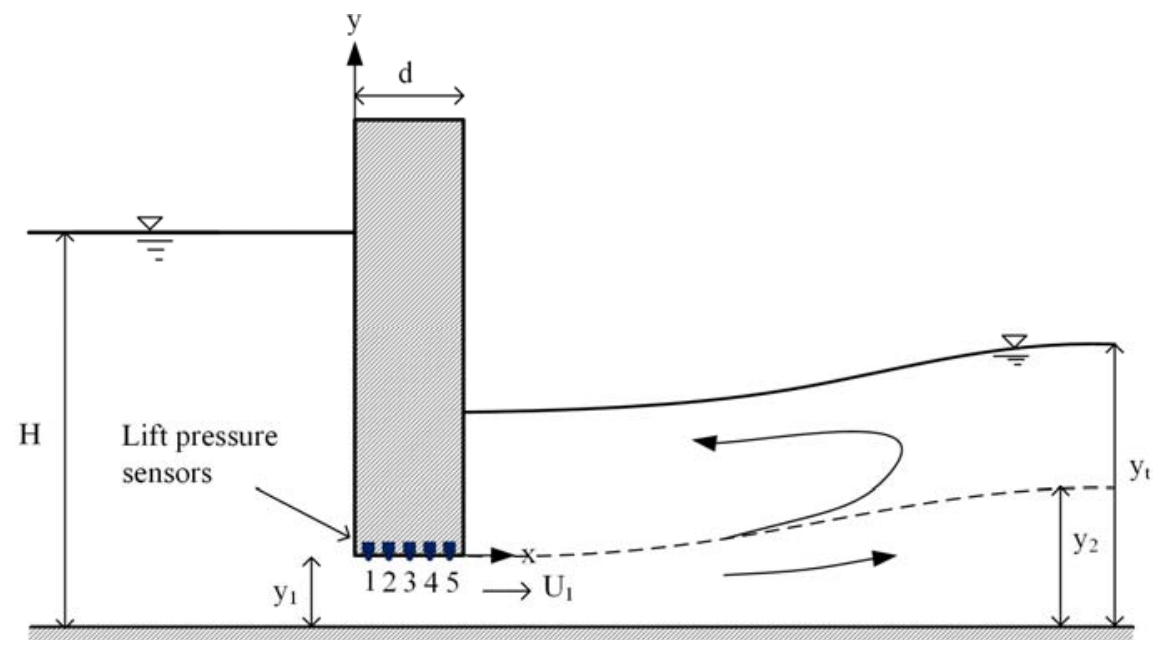

Figure 1: Schematic view of the SHJ downstream of a vertical gate.

Here $F_{1}$ is the Froude number below the gate, which is calculated as $F_{1}=U_{1} / \sqrt{g y_{1}}$ and $U_{1}$ is the average flow velocity below the gate. Schematic view of the SHJ downstream of the gate is shown in Fig. 1.

As depicted in Fig. 1, five pressure sensors were embedded at gate lip in order to observe spatial distribution of the lift pressures over the gate lip. Recirculating flow forms downstream of the gate and interacts with the mean flow to create vortical flow near the gate. Complex flow structure forming near the gate can result in significant hydrodynamic pressures on the control structure, which is required to be considered in design of vertical lift gates.

\section{EXPERIMENTAL SETUP}

In order to investigate the hydrodynamic pressures acting on the gate lip, experimental studies were conducted in a laboratory flume with $10 \mathrm{~m}$ long, $40 \mathrm{~cm}$ width and $60 \mathrm{~cm}$ height [3]. Sidewalls of the flume are transparent plexiglass and the bottom is stainless steel. The flow rate passing in the flume was adjusted using a valve on the feeding pipe and measured by ultrasonic flow meter (KATFlow 200) precisely. Gate opening can be adjusted vertically and fixed by the screws to provide a rigid gate condition (Fig. 2). Submergence ratio can be achieved using a tilting gate at the downstream of the flume to adjust desired tail water depth.

Five pressure sensors (Honeywell 24PC-EFA6G series) were embedded at the gate lip and pressure signals were collected from the stationary sensors using National Instrument data acquisition system (NI USB 6343) with the sampling frequency of $1000 \mathrm{~Hz}$. Experimental studies were conducted for different flow conditions as shown in Table 1 in order to observe the effect of Froude number and submergence factor on the results. A static calibration test was carried out in order to convert voltage signal to the pressure in Pascal. The following equation was determined from the static calibration test using different flow depths ranging from $2-36 \mathrm{~cm}$ in a cubic water tank

$$
V=0.0885 h+2.0313 \text {. }
$$

Here $V$ is the voltage output $(\mathrm{mV})$ and $h$ is the water depth $(\mathrm{mm})$. 
Moving-average filtering is applied to the raw data in order to eliminate possible effects arising from the noise in the electricity infrastructure since the collected voltage is highly sensitive to the small changes in voltage. Comparison of the raw and filtered data is shown in Fig. 3, which were measured at sensor 4 for the Case 3. It can be seen from the figure that the present filtering procedure can successfully filter the excessive pressure measurements due to the noise effects.

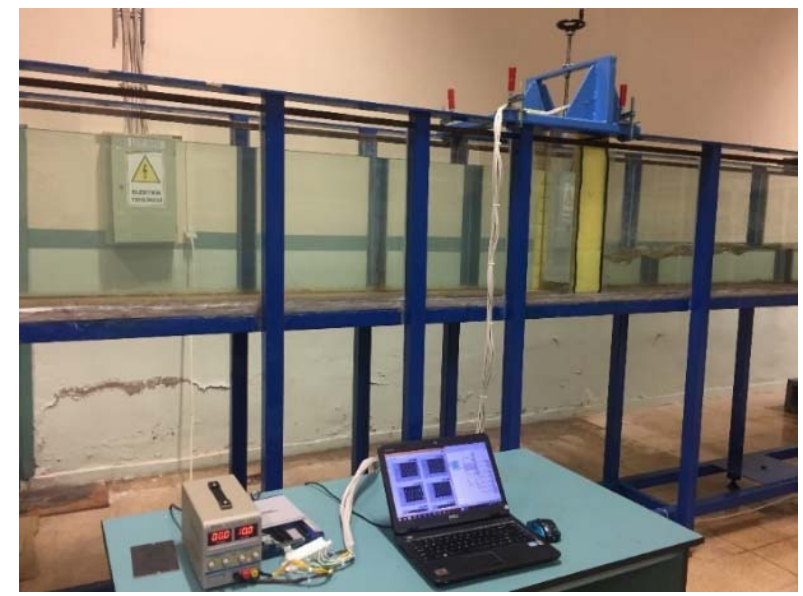

Figure 2: Experimental setup and measurement.

Table 1: Flow parameters.

\begin{tabular}{ccccccccc}
\hline Case & $\mathrm{Q}\left(\mathrm{m}^{3} / \mathrm{s}\right)$ & $\mathrm{H}(\mathrm{m})$ & $\mathrm{y}_{1}(\mathrm{~m})$ & $\mathrm{U}_{1}(\mathrm{~m} / \mathrm{s})$ & $\mathrm{Fr}$ & $\mathrm{y}_{2}(\mathrm{~m})$ & $\mathrm{y}_{\mathrm{t}}(\mathrm{m})$ & $\mathrm{S}$ \\
\hline 1 & 0.028 & 0.55 & 0.045 & 1.556 & 2.341 & 0.128 & 0.231 & 0.8 \\
2 & 0.029 & 0.53 & 0.050 & 1.450 & 2.070 & 0.124 & 0.247 & 1.0 \\
3 & 0.031 & 0.60 & 0.050 & 1.550 & 2.213 & 0.133 & 0.267 & 1.0 \\
\hline
\end{tabular}

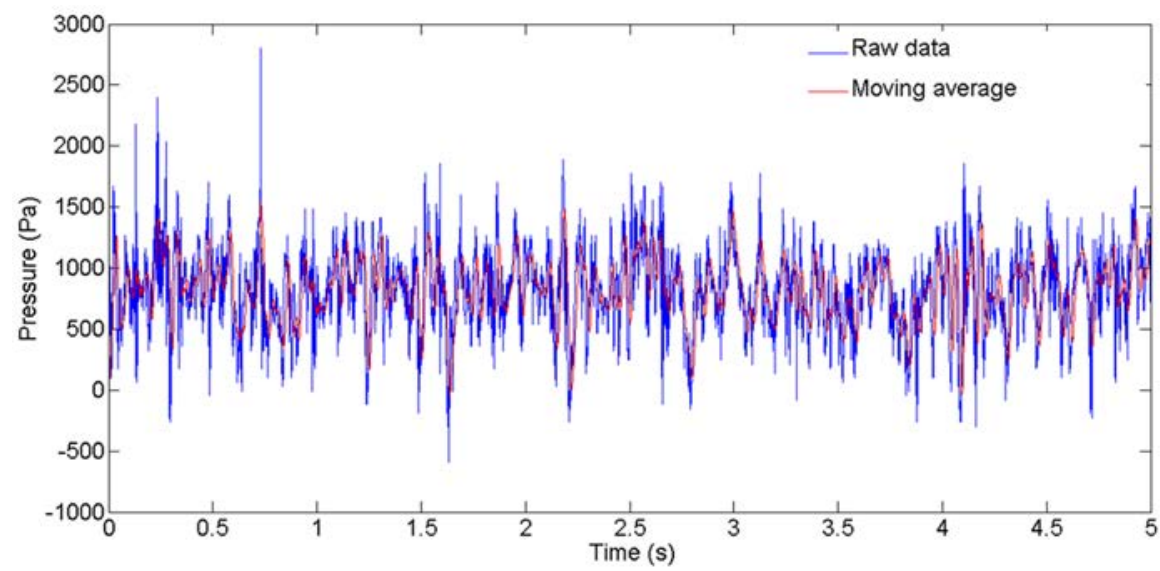

Figure 3: Comparison of the raw and filtered data obtained from the sensor 4 for Case 3. 
Time-averaged and instantaneous pressures acting on the gate lip are evaluated using the following definitions of mean pressure and instantaneous pressure coefficients [4], [5]

$$
\begin{gathered}
C_{\bar{p}}=\frac{\left(H-\frac{\bar{p}}{\gamma}\right)}{\frac{U_{1}^{2}}{2 g}}, \\
C_{p^{\prime}}=\frac{\sqrt{\overline{p^{\prime 2}}}}{\frac{1}{2} \rho U_{1}^{2}} \frac{y_{t}}{H},
\end{gathered}
$$

where $H$ is the upstream water depth, $\bar{p}$ is the mean pressure and $\rho$ is the density of the water. By definition, high pressure coefficient corresponds to low pressure magnitude.

\section{RESULTS AND DISCUSSION}

Time variations of lift pressures are demonstrated in Fig. 4 for each experimental case. Remember that the submergence factor is identical in cases 2 and 3. Froude number is much effective on the pressure fluctuations at sensors 1 and 5 since the separation of the flow from the leading edge of the gate and reattachment on the trailing edge can significantly increase the pressure fluctuations at those locations. High flow velocities at the entrance of the gate opening reduced the pressure magnitudes as seen in Fig. 4(a). Effects of the Froude number and submergence factor are almost absent at sensor 4, which is due to fact that this point of location is not as sensitive as the corner locations to the separation effects.

Variations of mean and instantaneous pressure coefficients are shown in Fig. 5 for each case. Mean pressures are low near the leading edge of the gate due to high momentum of the flow at the entrance. The location of the high mean pressure coefficient near $x / d \approx 0.51$ corresponds to the stagnation point just downstream of the midway of the gate since the separated flow impinges to the gate lip and increases pressure significantly. Distribution of the instantaneous pressure coefficient over the gate lip shows a trend line for the case 2 and
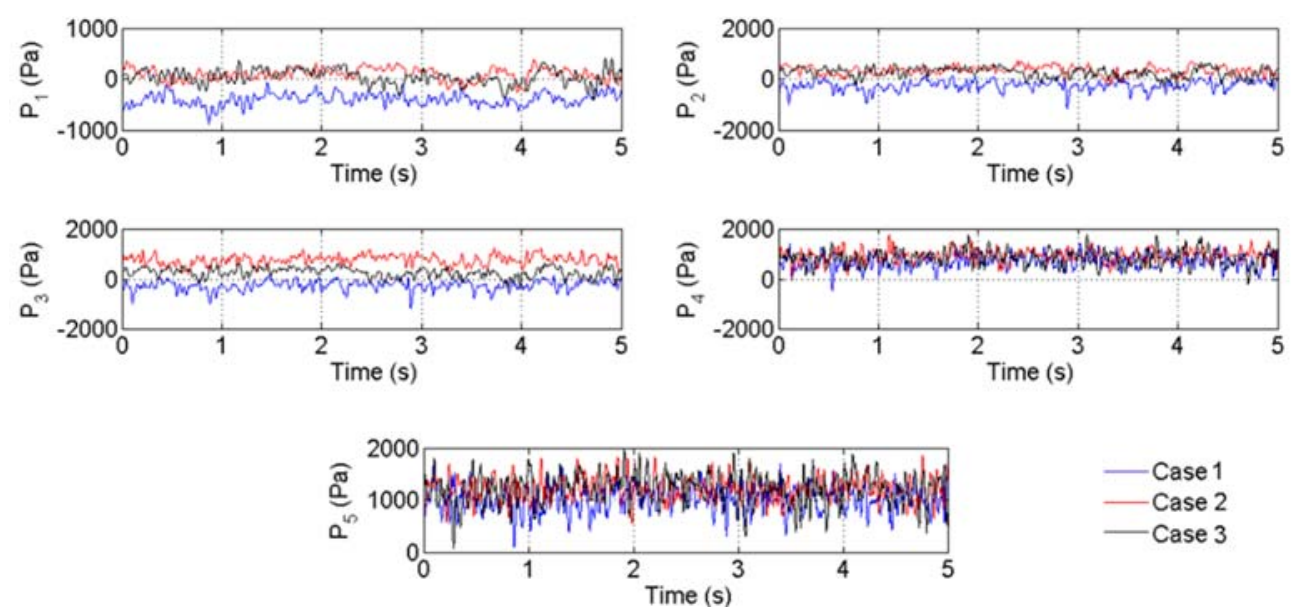

Figure 4: Pressure fluctuations at lift sensors for each case. 
drops suddenly at $x / d \approx 0.68$, which may be due to the interaction of the backward flow with mean flow as can be seen for all cases in Fig. 5(b). It should be noted that the spatial coordinates in the figure are non-dimensionalized with respect to thickness of the gate.

In order to determine the dominant frequency in the variation of pressures on the gate, power spectrum density (PSD) analysis is performed using Hamming windows with $n=2^{10}$ segment lengths and 50\% overlap in Matlab. Pressure fluctuations are expected to be high at the leading and trailing edges of the gate due to the separation effects, as also observed in the previous plots.

Frequency spectra of the lift pressures were computed and shown in Fig. 6 for sensors 1 and 5, at which the pressure fluctuations are expected to be high. The above power spectra reveals a dominant frequency of $15 \mathrm{~Hz}$ and the magnitude of the peak increases as the Froude number increases. The difference between cases 2 and 3 shows that the Froude number significantly affects the power spectra even for the same constant submergence factors. Moreover, the power spectra is almost independent of the submergence factor as observed from the comparison of the results of cases 1 and 3. Although the magnitude of the spectra at sensor 5 is greater than at sensor 1, the peak at sensor 1 is more apparent compared to the sensor 5. Fig. 6(b) clearly shows that the slope of the spectra is independent of the flow conditions.

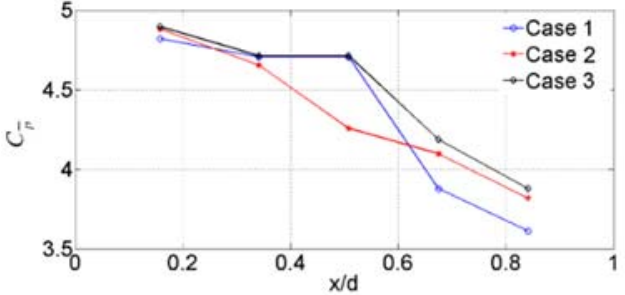

(a)

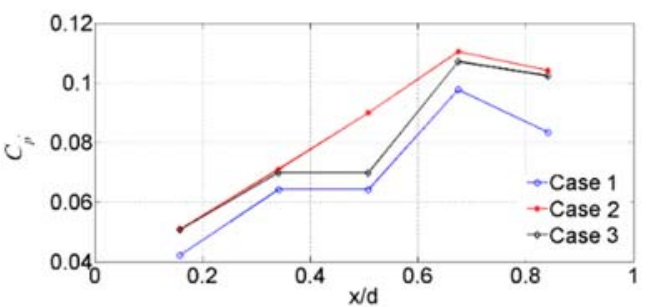

(b)

Figure 5: Distribution of the pressure coefficients over the gate lip. (a) Mean; (b) Instantaneous pressure coefficients.

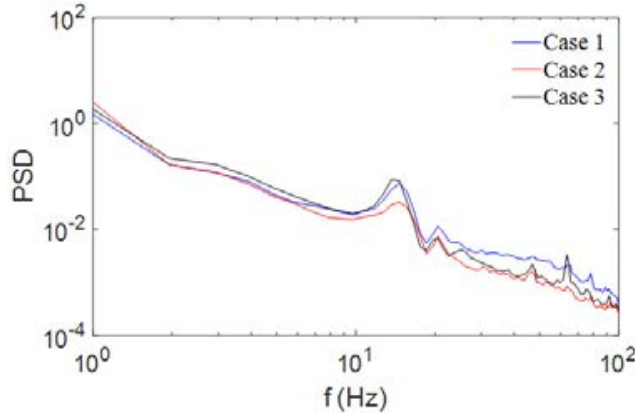

(a)

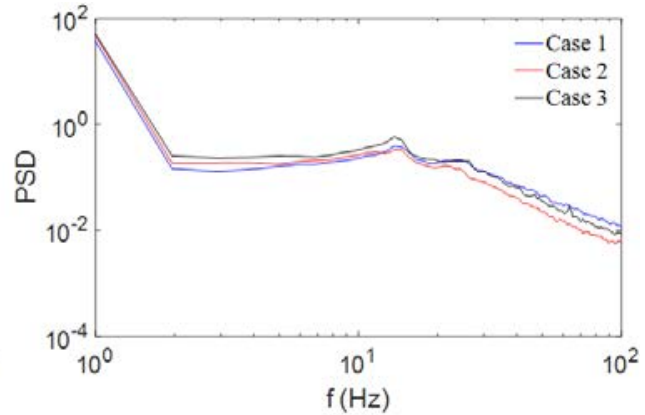

(b)

Figure 6: Spectra of lift pressure fluctuations. (a) Sensor 1; (b) Sensor 5. 


\section{CONCLUSIONS}

Experimental studies were conducted in the laboratory for the investigation of lift pressure acting on the lip of the gate subjected to the submerged hydraulic jump. Different flow conditions are provided in the laboratory flume in order to see the effects of Froude number and submergence factor on the results. Pressure fluctuations were measured simultaneously using five pressure sensors embedded at different locations on the gate lip. Time variations of pressure fluctuations over the gate lip show that the fluctuation components are high at the leading and trailing edges of the gate due to the separation effects below the gate. Distribution of mean pressure coefficient over the gate lip shows the stagnation point at approximately $x / d \approx 0.51$. The power spectra reveals a dominant frequency of $15 \mathrm{~Hz}$ near the leading edge of the gate and tends to decrease with the same slope for all cases.

\section{ACKNOWLEDGEMENT}

This work was supported by the Scientific and Technological Research Council of Turkey (TUBITAK) under Grant no. 216M521.

\section{REFERENCES}

[1] Wu, S. \& Rajaratnam, N., Free jumps, submerged jumps and wall jets. Journal of Hydraulic Research, 33(2), pp. 197-212, 1995.

[2] Rajaratnam, N., Hydraulic jumps. Advance in Hydrosciences, vol. 4, ed. V.T. Chow, Academic Press: New York, pp. 198-280, 1967.

[3] Demirel, E., Measured and simulated flow downstream of the submerged sluice gate. Water and Environment Journal, 29(3), pp. 446-455, 2015.

[4] Bhargava, V.P. \& Narasimhan, S., Pressure fluctuations on gates. Journal of Hydraulic Research, 27(2), pp. 215-231, 1989.

[5] Thang, N.G \& Naudascher, E., Vortex-excited vibration of underflow gates. Journal of Hydraulic Research, 24(2), pp. 133-151, 1986. 